\title{
EDITORIAL
}

\section{Virtual networking for microbiologists}

\author{
The advent of Web 2.0 applications, which enable information sharing and virtual \\ networking, could revolutionize science. But are microbiologists taking advantage?
}

Thousands of microbiologists will soon be converging on Boston for the 108th General Meeting of the American Society for Microbiology (ASM), the largest microbiology conference of the year. Networking is an essential part of the conference experience, but what opportunities are there for those who cannot make it to many, or even any, meetings? Can Web 2.0 applications enable scientists that do not have the time or money to attend meetings to reap the benefits of networking, and do Web 2.0 applications have a place in both our social and work lives? In the spirit of networking, we have taken a look at selected virtual networking resources that might be useful for microbiologists.

Wiki software enables users to create and edit web pages via a web browser, and the use of laboratory wiki's is on the increase among scientists. A laboratory wiki can be a great way to store and share protocols, results, presentations, posters and partly written papers online, as well as being useful for organizing meetings and keeping track of busy group schedules. With a laboratory wiki online, the days of leafing through illegible notebooks to hunt for old protocols could, thankfully, be over. Another benefit of wikis is that collaborating researchers can share data without having to resort to emailing bulky files to and fro. Reassuringly, wiki technology is safeguarded with user passwords, which means that data are private. For those who want to make some, or all, of the content on their laboratory wikis open for all to see, there is OpenWetWare. Of the 129 laboratories that are up and running on OpenWetWare, several (including those of McKinney, Keymer and French) are microbiology laboratories.

Researchers in the physical sciences have led the way

A laboratory

wiki can be a

great way to

store and share

protocols, results,

presentations,

posters and

partly written

papers online... in data sharing with arXiv, a pre-print server that was established in 1991. Life scientists have traditionally been a more secretive bunch and not everyone thinks that open data-sharing can work, especially in competitive fields. Nature Precedings is a new pre-print server for life scientists that currently has $>30$ entries listed under microbiology. Using Nature Precedings is one way to get feedback from the community on your preliminary findings. Only time will tell if microbiologists and other life scientists decide to embrace data sharing, which might have the potential to accelerate research.
Most Ph.D. students and postdoctoral fellows will probably be familiar with social networking sites, such as Facebook and Bebo. A quick search of Facebook reveals 205 microbiology groups, many of which electronically unite students and researchers that either love or hate microbiology, with discussion topics that range from 'what's your favourite microbe?' to 'how do I extract chromosomal DNA?' Nature Network is a social networking tool designed for scientists that is gradually building up active users and also has several microbiology groups. One advantage of such sites is that in addition to allowing users to keep in touch with a network of contacts worldwide, they can also host various 'blogs'.

Finding the time to write and read blogs could stimulate collaborations or open up new career opportunities in science writing and education, but how many microbiologists actually write blogs? In the United Kingdom, the only microbiology academic who blogs on a regular basis is Alan Cann from the University of Leicester. His blog on new and exciting microbiology research, aptly named MicrobiologyBytes, has been particularly successful, with 300,000 page views over the past 18 months and 750 comments on 468 posts (Alan filters out all spam comments or 'splogs'). He also blogs about science in general on Science of the Invisible, and his enthusiasm for new technologies has led him to divert his energy into a non-laboratory-based career researching the use of online resources for education. Other active microbiology 'bloggers' include Moeslio Schaechter, whose blog Small Things Considered is hosted by the ASM, César Sánchez, whose blog Twisted Bacteria has a particular focus on actinomycetes and Ed Rybicki, whose blog ViroBlogy concentrates on virology. Some other bloggers, including microbiologist Rosie Redfield, focus more on their own research to provide an insight into the everyday lives of research scientists.

It is now even possible to attend conferences without leaving your desk: the first ever International Online Medical Conference was held on 10-11 May. But how useful will Web 2.0 applications be in your research? We welcome your views, so this Editorial will be posted for comment at http://blogs.nature.com/nautilus/ 2008/05/microbiologists_and_the_social.html. 